\section{Comparison of intravenous aminophylline and salbutamol in severe asthma}

Severe asthma is the most common respiratory emergency treated in casualty departments of most hospitals. Intravenous aminophylline has been the mainstay of bronchodilator treatment for several years, although sudden death may occur after rapid administration, ${ }^{12}$ and it may be cardiotoxic. ${ }^{3}$ We thought that a selective adrenoceptor agonist might therefore be worthy of trial, and we report here our results with single- and double-blind comparative trials of intravenous aminophylline and salbutamol on 50 patients with severe asthma.

\section{Patients, methods, and results}

All patients gave informed consent to the studies, and treatment was allocated at random. In the single-blind study eight and 10 patients respectively received aminophylline $250 \mathrm{mg}$ and salbutamol $200 \mu \mathrm{g}$, and in the double-blind study 15 and 17 patients respectively received these two drugs. All patients had peak expiratory flow rates (PEFR) of less than $165 \mathrm{l} / \mathrm{min}$. Baseline observations of pulse rate and peak expiratory flow rate (measured with a Wright peak flow meter) were made at five-minute intervals 10 minutes before the injections.

In the single-blind study aminophylline was given over 15 minutes, while salbutamol was given in a bolus for up to one minute. In the double-blind study, however, both drugs were administered in not less than 15 minutes.

Pulse rate and PEFR were observed at five-minute intervals for 40 minutes. Asthmatics with more severe disease whose pulses were difficult to count because of pulsus paradoxus were subjected to electrocardiogram monitoring. Mean age and baseline pulse rate and PEFR were comparable in the aminophylline and salbutamol groups.

The table shows the mean peak increase or decrease of pulse rate and PEFR after treatment in the two study groups. The pulse rate tended to be higher in the single-blind group after salbutamol, and the bronchodilator effect was enhanced by bolus administration within one minute. In the double-blind study, however, there was no significant difference between either of the drugs in peak increase or decrease of pulse rate and PEFR.

\section{Comment}

The doses of aminophylline and salbutamol used may not have been optimal. Although our double-blind study showed no statistically significant differences in pulse rate and PEFR between aminophylline and salbutamol, the comparative success of salbutamol in the singleblind study gives it a distinct advantage over aminophylline in a busy casulaty or emergency department. The administration of salbutamol in a one-minute bolus intravenously increased the bronchodilator effect at every five-minute interval and was statistically significant at five and 20 minutes in the single-blind study (see table).

A therapeutic plasma level of aminophylline may be achieved by giving an intravenous loading dose of $5.6 \mathrm{mg} / \mathrm{kg}$ over 15 to 30 minutes. ${ }^{3}$ This is a long time to spend on an intravenous injection in a busy casualty or emergency department, especially in areas with inadequate provision of medical personnel. Thus there is a case for more extensive use of intravenous salbutamol to discover its optimum dosage and mode of administration. The results of the one-minute bolus administration of intravenous salbutamol in this study and elsewhere ${ }^{4}$ are encouraging, and if confirmed by other investigators this method of administration might represent a modest advance in bronchodilator treatment.

We thank Dr K O Odusote, Mrs K O Williams, and Mr J Adepoju for help with this study.

${ }^{1}$ Merrill, G A, Fournal of the American Medical Association, 1943, 123, 1115.

${ }^{2}$ Bresnick, E, Woodard, W K, and Sageman, C B, fournal of the American Medical Association, 1945, 136, 397.

${ }^{3}$ Lancet, 1973, 2, 950.

+ Fitchett, D H, McNicol, M W, and Riordan, J F, British Medical fournal, $1975,1,53$.

(Accepted 3 November 1976)

Respiratory Unit, Department of Medicine, College of Medicine of the University of Lagos, PMB 12003, Lagos, Nigeria

D FEMI-PEARSE, MD, FRCP, professor of medicine W O GEORGE, MB, BS, senior house officer

$S$ T ILECHUKWU, $M B$, BS, senior house officer

O O ELEGBELEYE, $M B, M R C P$, senior lecturer

A O AFONJA, MB, MRCP, senior lecturer

Mean peak changes $\pm S E$ in pulse rate and peak expiratory flow rate after intravenous aminophylline 250 mg and salbutamol $200 \mu g$. Decreases are prefixed by minus sign; other figures are increases

\begin{tabular}{|c|c|c|c|c|c|c|c|c|c|}
\hline & Time after injection (min): & 5 & 10 & 15 & 20 & 25 & 30 & 34 & 40 \\
\hline \multirow{6}{*}{$\begin{array}{l}\text { Single-blind } \\
\text { study } \\
\text { group }\end{array}$} & \multicolumn{9}{|c|}{ Pulse rate/min } \\
\hline & $\begin{array}{l}\text { Aminophylline }(n=8) \\
\text { Salbutamol }(n=10)\end{array}$ & $\begin{array}{r}9 \cdot 5: 4 \cdot 4 \\
13 \cdot 4 \pm 4 \cdot 0\end{array}$ & $\begin{array}{l}4 \cdot 0 \div 2 \cdot 1 \\
8 \cdot 6 \pm 3 \cdot 0\end{array}$ & $\begin{array}{r}0.25 \pm 2.9 \\
7.0 \pm 2.9\end{array}$ & $\begin{array}{rl}-1 \cdot 5 & 3 \cdot 6 \\
5 \cdot 4 & +3 \cdot 0\end{array}$ & $\begin{array}{r}-3 \cdot 2 \pm 2 \cdot 8 \\
2 \cdot 0 \pm 2 \cdot 5\end{array}$ & $\begin{array}{l}3 \cdot 2 \pm 2 \cdot 6 \\
1 \cdot 2 \pm 1 \cdot 4\end{array}$ & $\begin{array}{l}-4 \cdot 6 \pm 3 \cdot 2 \\
-1 \cdot 0 \pm 2 \cdot 2\end{array}$ & $\begin{array}{l}-4 \cdot 2 \pm 2 \cdot 3 \\
-1 \cdot 0 \pm 2 \cdot 5\end{array}$ \\
\hline & $P$ value & NS & NS & NS & NS & NS & NS & NS & NS \\
\hline & \multicolumn{9}{|c|}{ Peak expiratory flow rate (l/min) } \\
\hline & $\begin{array}{l}\text { Aminophylline }(n=8) \\
\text { Salbutamol }(n=10)\end{array}$ & $\begin{array}{l}12 \cdot 5 \pm 2 \cdot 8 \\
42 \cdot 5 \pm 7 \cdot 7\end{array}$ & $\begin{array}{l}18 \cdot 7 \pm 6 \cdot 3 \\
31 \cdot 0 \pm 6 \cdot 3\end{array}$ & $\begin{array}{l}20 \cdot 0 \div 5 \cdot 3 \\
45 \cdot 5 \pm 14 \cdot 7\end{array}$ & $\begin{array}{l}14 \cdot 4 \pm 4 \cdot 1 \\
43 \cdot 5 \pm 11 \cdot 3\end{array}$ & $\begin{array}{l}25 \cdot 0 \pm 9 \cdot 0 \\
42 \cdot 5 \pm 11 \cdot 9\end{array}$ & $\begin{array}{l}23 \cdot 7 \pm 9 \cdot 1 \\
44 \cdot 5 \pm 15 \cdot 2\end{array}$ & $\begin{array}{l}25 \cdot 6 \pm 7 \cdot 6 \\
47 \cdot 0 \pm 18 \cdot 1\end{array}$ & $\begin{array}{l}21 \cdot 2 \pm 7 \cdot 4 \\
48 \cdot 5 \pm 16 \cdot 1\end{array}$ \\
\hline & $P$ value & $\begin{array}{c}\mathrm{P}<0.005 \\
t=3.31\end{array}$ & NS & NS & $\begin{aligned} \mathrm{P} & <0 \cdot 05 \\
t & =2 \cdot 20\end{aligned}$ & NS & NS & NS & NS \\
\hline \multirow{6}{*}{$\begin{array}{l}\text { Double-blind } \\
\text { study } \\
\text { group }\end{array}$} & \multicolumn{9}{|c|}{ Pulse rate/min } \\
\hline & $\begin{array}{l}\text { Aminophylline }(n=15) \\
\text { Salbutamol }(n=17)\end{array}$ & $\begin{array}{l}4 \cdot 3 \pm 2 \cdot 4 \\
5 \cdot 2=2 \cdot 8\end{array}$ & $\begin{array}{l}1 \cdot 5 \pm 2 \cdot 2 \\
3 \cdot 9 \pm 2 \cdot 3\end{array}$ & $\begin{array}{r}-0.9 \pm 2.4 \\
1.5 \div 2.0\end{array}$ & $\begin{array}{l}-2 \cdot 9 \pm 2 \cdot 3 \\
-0.8 \pm 2 \cdot 1\end{array}$ & $\begin{array}{l}-1 \cdot 2 \pm 2 \cdot 2 \\
-1.9 \pm 1.9\end{array}$ & $\begin{array}{l}-3 \cdot 6 \pm 2 \cdot 7 \\
-3 \cdot 2 \pm 1 \cdot 6\end{array}$ & $\begin{array}{l}-3 \cdot 9 \pm 2 \cdot 6 \\
-3 \cdot 7 \pm 1 \cdot 7\end{array}$ & $\begin{array}{l}-3 \cdot 6 \pm 2 \cdot 5 \\
-4 \cdot 7 \pm 2 \cdot 0\end{array}$ \\
\hline & $P$ value & NS & NS & NS & NS & NS & NS & NS & NS \\
\hline & \multicolumn{9}{|c|}{ Peak expiratory rate (l/min) } \\
\hline & $\begin{array}{l}\text { Aminophylline }(n=15) \\
\text { Salbutamol }(n=17)\end{array}$ & $\begin{array}{l}28 \cdot 7 \pm 12 \cdot 1 \\
36 \cdot 5 \pm 10 \cdot 0\end{array}$ & $\begin{array}{l}36 \cdot 3 \pm 12 \cdot 6 \\
32 \cdot 1 \pm 8 \cdot 6\end{array}$ & $\begin{array}{l}34 \cdot 0=10 \cdot 3 \\
31 \cdot 5=9 \cdot 1\end{array}$ & $\begin{array}{l}33 \cdot 7 \pm 10 \cdot 2 \\
32 \cdot 4 \pm 8 \cdot 6\end{array}$ & $\begin{array}{l}31 \cdot 7 \pm 11 \cdot 9 \\
34 \cdot 4 \pm 9 \cdot 0\end{array}$ & $\begin{array}{l}29 \cdot 0 \pm 12 \cdot 0 \\
30 \cdot 9 \pm 9 \cdot 6\end{array}$ & $\begin{array}{l}31 \cdot 0 \pm 12 \cdot 6 \\
28 \cdot 2 \pm 9 \cdot 4\end{array}$ & $\begin{array}{l}29 \cdot 1 \pm 12 \cdot 8 \\
34 \cdot 1 \pm 10 \cdot 9\end{array}$ \\
\hline & $P$ value & NS & NS & NS & NS & NS & NS & NS & NS \\
\hline
\end{tabular}

NS $=$ Not significant. 